\title{
A Probe-Compensated Helicoidal NF-FF Transformation for Aperture Antennas Using a Prolate Spheroidal Expansion
}

\author{
Amedeo Capozzoli, ${ }^{1}$ Claudio Curcio, ${ }^{1}$ Giuseppe D'Elia, ${ }^{1}$ Flaminio Ferrara, ${ }^{2}$ \\ Claudio Gennarelli, ${ }^{2}$ Rocco Guerriero, ${ }^{2}$ and Angelo Liseno ${ }^{1}$ \\ ${ }^{1}$ Dipartimento di Ingegneria Biomedica, Elettronica e delle Telecomunicazioni, Università di Napoli Federico II, Via Claudio 21, \\ 80125 Napoli, Italy \\ ${ }^{2}$ Dipartimento di Ingegneria Elettronica ed Ingegneria Informatica, Università di Salerno, via Ponte Don Melillo, \\ Salerno, 84084 Fisciano, Italy
}

Correspondence should be addressed to Claudio Gennarelli, gennar@diiie.unisa.it

Received 10 October 2011; Accepted 23 January 2012

Academic Editor: Jeff Fordham

Copyright (c) 2012 Amedeo Capozzoli et al. This is an open access article distributed under the Creative Commons Attribution License, which permits unrestricted use, distribution, and reproduction in any medium, provided the original work is properly cited.

A new probe-compensated near-field-far-field (NF-FF) transformation for aperture antennas in a cylindrical scanning geometry is presented. Such a technique takes the advantage of the NF data acquisition made according to a very efficient sampling strategy along a helix and exploits a proper aperture field expansion based on the use of the prolate spheroidal wave functions (PSWFs), accounting for the a priori information on shape and size of the antenna under test. The unknown aperture field expansion coefficients of the PSWFs are evaluated from the acquired voltage samples by an inversion process using a regularized version of the singular value decomposition method. Experimental results on connected and disconnected radiating aperture antennas, including sum and difference patterns, show the effectiveness of the approach and, in particular, how it enables a serious reduction of the measurement points without impairing the FF estimation accuracy.

\section{Introduction}

As well known, near-field-far-field (NF-FF) transformations represent nowadays a widely used technique for antenna characterization as an alternative to measurements directly performed in the far zone of the radiating system [1-4]. Moreover, the pattern evaluation from NF measurements, carried out in a controlled environment (anechoic chamber), allows overcoming those drawbacks which make the measurement of the radiation pattern in a conventional FF range unpractical for electrically large antennas. In this framework, reducing the NF acquisition time is assuming an ever growing relevance for the antenna measurement community. In fact, such a time is currently very much greater than that needed to perform the corresponding NFFF transformation.

An effective way to reduce the measurement time in a NF cylindrical facility is the use of the innovative helicoidal scanning technique [5-9], which can be accomplished by means of continuous and synchronized movements of the positioning systems of the probe and antenna under test (AUT), as suggested by Yaccarino et al. in [10]. The technique in [5-9] relies on the "nonredundant sampling representations" of electromagnetic (EM) fields in [11] and uses optimal sampling interpolation (OSI) formulas to reconstruct the NF data required by the standard NF-FF transformation with cylindrical scanning $[12,13]$. The drastic time saving so achieved is due both to the use of continuous movements and to the significantly reduced number of needed NF data. The resulting NF-FF transformation has proved to be accurate, stable, and efficient. In particular, effective AUT modellings [7-9] have been used when dealing with antennas having a predominant dimension and allowed a further reduction of needed NF data with respect to the spherical AUT modelling $[5,6]$. However, when dealing with aperture antennas, such an additional a priori information available on the AUT can be conveniently exploited to provide the aperture field with an effective representation, suitably accounting for its shape and size as well as for its radiating features, thus further 
reducing the overall number of parameters to be sought for and improving the achievable accuracy [14-16].

Following the approach in [16], herewithin, we present an approach to the near-field characterization of aperture antennas in a cylindrical geometry based on the joint use of a proper helicoidal scanning and an efficient modal representation of the aperture field leading back the NF-FF transformation to an inversion step and a prompt subsequent radiation step. More in detail,

(i) the measurement voltages are acquired along a helicoidal curve determined according to the abovequoted approaches [6-9];

(ii) the prolate spheroidal wave functions (PSWFs) [17, 18], which are able to represent the radiative aperture field by a well-defined subspace, are employed in this paper;

(iii) the unknown aperture field expansion coefficients are evaluated from the measured voltages by an inversion process carried out by means of a regularized version of the singular value decomposition (SVD) approach (first step of the NF-FF transformation);

(iv) the radiated field is then evaluated by exploiting the link between the subspace spanned by the basis functions used to represent the aperture field and their Fourier transforms (second step of the NF-FF transformation).

It should be noticed that previous efforts have been already pursued in the literature to use the SVD in NFFF transformations [19]. However and as well known [14], the sinc sampling representation of the plane wave spectrum (PWS) radiated by the source is significantly suboptimal as compared to the PSWFs representation.

In contrast with [16], the method is here presented in its complete formulation accounting also for the probe compensation, and the performance is evaluated against experimental tests involving different aperture antenna configurations, enhancing key aspects related, among others, to disconnected aperture domains and field polarization. The experimental results show the effectiveness of the technique and how much it enables to reduce the number of measurement points, leaving the performance unchanged.

The paper is organized in six sections and two appendices.

Section 2 describes the sampling strategy in [6-9] for the helicoidal scanning using effective AUT modellings. Section 3 introduces the PSWF-based representation [15, $16]$ to be exploited in the case of connected and disconnected rectangular apertures. Section 4 describes the probecompensated inversion technique using the SVD method and the radiation procedure (steps 1 and 2). In Section 5, the experimental results obtained via the proposed technique are compared with those relevant to the helicoidal NF-FF transformations $[6,8]$ and to the standard NF-FF cylindrical transformation $[12,13]$. Finally, in Section 6 , conclusions are drawn and future developments sketched.

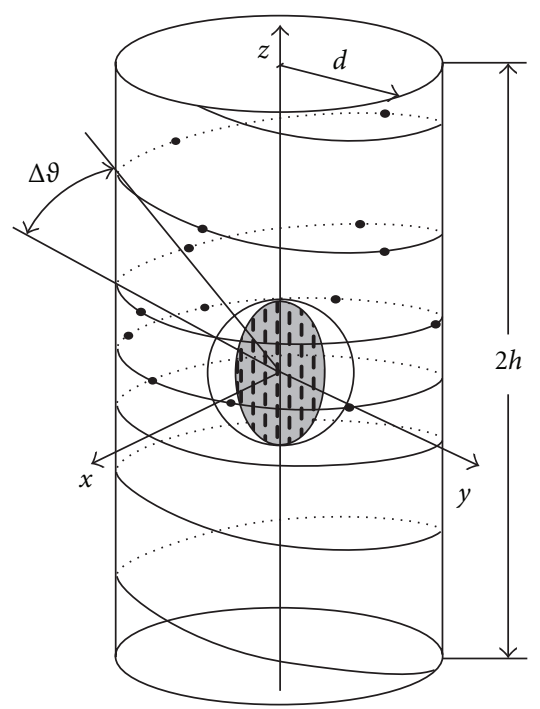

Figure 1: Helicoidal scanning.

\section{Helicoidal Sampling Strategy}

The voltage sampling representation on a cylinder from its samples acquired along a helix (see Figure 1) is summarized in the following.

Let us consider an AUT, enclosed in a convex domain bounded by a surface $\Sigma$ with rotational symmetry and a nondirective probe scanning a proper helix lying on a cylinder of radius $d$. The spherical coordinate system $(r, \vartheta, \varphi)$ is adopted to denote an observation point $P$. Since the voltage measured by a nondirective probe has the same effective spatial bandwidth of the AUT field [20], the "nonredundant sampling representation" of EM fields [11] can be applied to it. Accordingly, when dealing with the voltage representation on an observation curve $C$, it is convenient to adopt a proper analytical parameterization $\underline{r}=\underline{r}(\eta)$ for describing $C$ and to introduce the "reduced voltage"

$$
\tilde{V}(\eta)=V(\eta) \mathrm{e}^{j \psi(\eta)},
$$

where $V(\eta)$ is the measured probe voltage and $\psi(\eta)$ is a proper phase function. The band limitation error, occurring when $\widetilde{V}(\eta)$ is approximated by a spatially bandlimited function, becomes negligible as the bandwidth exceeds a critical value $W_{\eta}$ [11]. Therefore, such an error can be effectively controlled by choosing a bandwidth equal to $\chi^{\prime} W_{\eta}$, where $\chi^{\prime}>1$ is the bandwidth enlargement factor.

The unified theory of spiral scannings for nonspherical antennas [21], obtained by paralleling the corresponding procedure based on the spherical AUT modelling [22], allows the development of the voltage representation on the cylinder from a minimum number of its samples on the helix. To this end, it is necessary

(a) to choose the step of the helix coincident with that needed for the interpolation along a generatrix;

(b) to determine an effective sampling representation along the helix. 
In particular, according to [21], the bandwidth $W_{\eta}$ and parameterization $\eta$ relevant to a generatrix and the corresponding phase function $\psi$ are given by

$$
\begin{aligned}
W_{\eta} & =\frac{\beta \ell^{\prime}}{2 \pi}, \\
\psi & =\frac{\beta}{2}\left[R_{1}+R_{2}+s_{1}^{\prime}-s_{2}^{\prime}\right], \\
\eta & =\frac{\pi}{\ell^{\prime}}\left[R_{1}-R_{2}+s_{1}^{\prime}+s_{2}^{\prime}\right],
\end{aligned}
$$

where $\beta$ is the wavenumber, $\ell^{\prime}$ is the length of $C^{\prime}$ (intersection curve between the meridian plane and $\Sigma$ ), $s_{1,2}^{\prime}$ are the arclength coordinates of the two tangency points $P_{1,2}$ between the cone of vertex at the observation point $P$ and $C^{\prime}$, and $R_{1,2}$ are the distances from $P$ to $P_{1,2}$.

The helix can be obtained by projecting on the scanning cylinder a proper spiral wrapping the surface $\Sigma$. The projection is obtained via the curves at $\eta=$ const [21]. The helix step, determined by two consecutive intersections with a generatrix, is equal to the sample spacing $\Delta \eta=2 \pi /\left(2 N^{\prime \prime}+\right.$ 1) relevant to a generatrix. Note that $N^{\prime \prime}=\operatorname{Int}\left(\chi N^{\prime}\right)+1$ $(\operatorname{Int}(x)$ denotes the integer part of $x), N^{\prime}=\operatorname{Int}\left(\chi^{\prime} W_{\eta}\right)+1$, and $\chi>1$ is an oversampling factor [11], which controls the truncation error. Accordingly, the parametric equations of the helix, when imposing its passage through a given point $Q_{0}$ of the generatrix at $\varphi=0$, are

$$
\begin{aligned}
& x=d \cos \left(\phi-\phi_{s}\right), \\
& y=d \sin \left(\phi-\phi_{s}\right), \\
& z=d \cot [\theta(\eta)],
\end{aligned}
$$

where in $\phi$ is the parameter describing the helix, $\phi_{s}$ is the value of $\phi$ at $Q_{0}$, and $\eta=k \phi, k$ being a parameter related to the helix step by $k=\Delta \eta / 2 \pi=1 /\left(2 N^{\prime \prime}+1\right)$.

Let us now denote with $\xi$ and $\gamma$ the optimal parameter and the phase function, respectively, to get a nonredundant representation along the helix. They can be again determined according to [21]. In particular, $\xi$ is $\beta / W_{\xi}$ times the arclength of the projecting point on the spiral wrapping $\Sigma$ and $\gamma$ coincides with the phase function $\psi$ relevant to a generatrix, where $W_{\xi}$ is equal to $\beta / \pi$ times the length of the spiral wrapping $\Sigma$ from pole to pole.

By exploiting the above results, the reduced voltage at any point $Q$ of the helix can be recovered via the OSI expansion [21]:

$$
\tilde{V}(\xi)=\sum_{m=m_{0}-p+1}^{m_{0}+p} \tilde{V}\left(\xi_{m}\right) \Omega_{M}\left(\xi-\xi_{m}\right) D_{M^{\prime \prime}}\left(\xi-\xi_{m}\right),
$$

where $2 p$ is the number of retained samples $\tilde{V}\left(\xi_{m}\right), m_{0}=$ $\operatorname{Int}\left[\left(\xi-\xi\left(\phi_{s}\right)\right) / \Delta \xi\right]$ is the index of the sample nearest (on the left) to $Q$, and

$$
\xi_{m}=\xi\left(\phi_{s}\right)+m \Delta \xi=\xi\left(\phi_{s}\right)+\frac{2 \pi m}{\left(2 M^{\prime \prime}+1\right)}
$$

with $M^{\prime \prime}=\operatorname{Int}\left(\chi M^{\prime}\right)+1$ and $M^{\prime}=\operatorname{Int}\left(\chi^{\prime} W_{\xi}\right)+1$. Moreover,

$$
\begin{aligned}
D_{M^{\prime \prime}}(\xi) & =\frac{\sin \left[\left(2 M^{\prime \prime}+1\right) \xi / 2\right]}{\left(2 M^{\prime \prime}+1\right) \sin (\xi / 2)}, \\
\Omega_{M}(\xi) & =\frac{T_{M}\left[2 \cos ^{2}(\xi / 2) / \cos ^{2}(\bar{\xi} / 2)-1\right]}{T_{M}\left[2 / \cos ^{2}(\bar{\xi} / 2)-1\right]}
\end{aligned}
$$

are the Dirichlet and Tschebyscheff sampling functions, respectively, $T_{M}(\cdot)$ being the Tschebyscheff polynomial of degree $M=M^{\prime \prime}-M^{\prime}$ and $\bar{\xi}=p \Delta \xi$.

Expansion (4) can be properly employed to evaluate the voltage at any point $P$ on the cylinder and, in particular, at those required to carry out the classical NF-FF transformation with cylindrical scanning $[12,13]$. Indeed, it allows the evaluation of the "intermediate" samples, that is, the voltages at the intersection points of the helix with the generatrix through $P$. Once these samples have been determined, the reduced voltage at $P$ can be reconstructed via the following OSI expansion [21]:

$$
\tilde{V}(\eta(9), \varphi)=\sum_{n=n_{0}-q+1}^{n_{0}+q} \tilde{V}\left(\eta_{n}\right) \Omega_{N}\left(\eta-\eta_{n}\right) D_{N^{\prime \prime}}\left(\eta-\eta_{n}\right),
$$

wherein $N=N^{\prime \prime}-N^{\prime}, n_{0}=\operatorname{Int}\left[\left(\eta-\eta_{0}\right) / \Delta \eta\right], 2 q$ is the number of the retained intermediate samples $\tilde{V}\left(\eta_{n}\right)$, and

$$
\eta_{n}=\eta_{n}(\varphi)=\eta\left(\phi_{s}\right)+k \varphi+n \Delta \eta=\eta_{0}+n \Delta \eta .
$$

When the AUT is a quasispherical antenna, it is convenient to consider as surface $\Sigma$ the smallest sphere of radius $a$ enclosing it. In such a case, $\ell^{\prime}=2 \pi a$ and $\eta=\vartheta$ so that $W_{\eta}=\beta a$ and the curves at $\eta=$ const become radial lines [22]. Moreover,

$$
\gamma=\psi=\beta \int_{0}^{r} \sqrt{1-\frac{a^{2}}{r^{\prime 2}}} \mathrm{~d} r^{\prime}=\beta \sqrt{r^{2}-a^{2}}-\beta a \cos ^{-1}\left(\frac{a}{r}\right),
$$

and the parameter $\xi$ describing the helix can be expressed in closed form as

$$
\xi=\frac{\beta a}{W_{\xi}} \int_{0}^{\phi} \sqrt{k^{2}+\sin ^{2} k \phi^{\prime}} \mathrm{d} \phi^{\prime},
$$

wherein

$$
W_{\xi}=\frac{\beta a}{\pi} \int_{0}^{\left(2 N^{\prime \prime}+1\right) \pi} \sqrt{k^{2}+\sin ^{2} k \phi^{\prime}} \mathrm{d} \phi^{\prime} .
$$

In the case of elongated antennas, an effective modelling is obtained by choosing $\Sigma$ coincident with the smallest prolate ellipsoid with major and minor semiaxes equal to $a$ and $b$. Accordingly, the bandwidth $W_{\eta}$, the parameterization $\eta$, and the phase function $\psi$ are $[7,8]$

$$
W_{\eta}=\left(\frac{4 a}{\lambda}\right) E\left(\frac{\pi}{2} \mid \varepsilon^{2}\right),
$$




$$
\begin{gathered}
\eta=\frac{\pi}{2}\left[1+\frac{E\left(\sin ^{-1} u \mid \varepsilon^{2}\right)}{E\left(\pi / 2 \mid \varepsilon^{2}\right)}\right], \\
\gamma=\psi=\beta a\left[v \sqrt{\frac{v^{2}-1}{v^{2}-\varepsilon^{2}}}-E\left(\cos ^{-1} \sqrt{\frac{1-\varepsilon^{2}}{v^{2}-\varepsilon^{2}}} \mid \varepsilon^{2}\right)\right],
\end{gathered}
$$

wherein $\lambda$ is the wavelength and $u=\left(r_{1}-r_{2}\right) / 2 f, \mathrm{v}=\left(r_{1}+\right.$ $\left.r_{2}\right) / 2 a$ are the elliptic coordinates, $r_{1,2}$ being the distances from observation point $P$ to the foci of the ellipse $C^{\prime}$ and $2 f$ its focal distance. In the above relations, $\varepsilon=f / a$ is the eccentricity of $C^{\prime}$ and $E(\cdot \mid \cdot)$ denotes the elliptic integral of second kind.

It is worth noting that, in such a case, the projecting curves are hyperbolas confocal to the ellipse $C^{\prime}$. Moreover, the parameter $\xi$, which is $\beta / W_{\xi}$ times the arclength of the projecting point on the spiral wrapping the prolate ellipsoid, and the related bandwidth $W_{\xi}$ must be numerically evaluated.

\section{Aperture Field Representation}

It is noted that the rationale of the technique holds for an arbitrary number of disconnected, arbitrarily shaped apertures, as well as for an arbitrarily polarized aperture field. However, in this paper, the approach will be presented in the case of two rectangular-shaped apertures. For each aperture, a separate PSWF expansion is given and the general case of an arbitrarily polarized aperture field will be considered (see Section 4). Furthermore, for the sake of simplicity, when dealing with the unknowns-to-data relation, the attention will be subsequently focused to the two cases of interest for this paper, namely, to tangential aperture field having "vertical" or "horizontal" polarization (in the meaning specified below).

Let us now formally work out the representation. As mentioned, two rectangular apertures, $A_{1}$ and $A_{2}$, are considered, both $2 a_{a p} \times 2 b_{a p}$ sized, with centres at a distance $d_{a}$. The radiating system is centred in the Oxyz reference system (see Figure 2). We suppose that the aperture field $\underline{E}_{a}\left(x_{i}, z_{i}\right)$ can be either "vertically" polarized, that is, $\underline{E}_{a}\left(x_{i}, z_{i}\right)=$ $E_{a_{y}}\left(x_{i}, z_{i}\right) \hat{i}_{y}+E_{a_{z}}\left(x_{i}, z_{i}\right) \hat{i}_{z}$, or "horizontally" polarized, that is, $\underline{E}_{a}\left(x_{i}, z_{i}\right)=E_{a_{x}}\left(x_{i}, z_{i}\right) \hat{i}_{x}+E_{a_{y}}\left(x_{i}, z_{i}\right) \hat{i}_{y}$. As well known, the field for $y>0$ is provided by the knowledge of the projection of the aperture field $\underline{E}_{a}\left(x_{i}, z_{i}\right)$ onto the aperture plane $x z$. Accordingly, in the former case, the field is provided by the knowledge of the function $E_{a_{z}}\left(x_{i}, z_{i}\right)$, whereas, in the latter case, by the knowledge of $E_{a_{x}}\left(x_{i}, z_{i}\right)$. Therefore, just a representation either for $E_{a_{z}}\left(x_{i}, z_{i}\right)$ or $E_{a_{x}}\left(x_{i}, z_{i}\right)$ is in order.

Following [14], it is convenient to represent the tangential components of the field on the disconnected aperture $A=A_{1} \cup A_{2}$ by basis functions having support on $A$ and spectrally limited to the visible region of the radiative field. Accordingly, each of the two relevant aperture fields is represented by means of PSWF expansions, involving different expansion coefficients, that is,

$$
\begin{aligned}
& E_{a_{t}}\left(x_{i}, z_{i}\right) \\
& =\left\{\begin{array}{c}
\sum_{p=1}^{P} \sum_{q=1}^{Q} g_{p q}^{(1)} \Phi_{p}\left[c_{x}, x_{i}\right] \Phi_{q}\left[c_{z}, z_{i}-\frac{d_{a}}{2}\right], \\
\text { if }\left(x_{i}, z_{i}\right) \in A_{1}, \\
\sum_{p=1}^{P} \sum_{q=1}^{Q} g_{p q}^{(2)} \Phi_{p}\left[c_{x}, x_{i}\right] \Phi_{q}\left[c_{z}, z_{i}+\frac{d_{a}}{2}\right], \\
\text { if }\left(x_{i}, z_{i}\right) \in A_{2},
\end{array}\right.
\end{aligned}
$$

where $\Phi_{i}\left[c_{w}, w\right]$ is the $i$ th, 1D PSWF with "space-bandwidth product" $c_{w}[17,18], c_{x}=a_{a p} u^{\prime}, c_{z}=b_{a p} v^{\prime}$, and $u^{\prime}, v^{\prime}$ locate the spectral region of interest [14], as $u^{\prime} \leq \beta$ and $v^{\prime} \leq \beta$. In (15), $P=\operatorname{Int}\left[4 a_{a p} / \lambda\right], Q=\operatorname{Int}\left[4 b_{a p} / \lambda\right]$, and, in our framework, the $g_{p q}^{(1)} s$ and $g_{p q}^{(2)} s$ are the expansion coefficients to be considered as unknowns and to be determined from the knowledge of the voltages delivered by the probe when scanning the measurement curve. Moreover, the 2D PSWFs have separate support over the two apertures $A_{1}$ and $A_{2}$ and $P \times Q$ represents the number of $2 \mathrm{D}$ PSWFs required by each aperture.

\section{NF-FF Transformation Technique}

In this section, we first derive the link between the unknown expansion coefficients $g_{p q}^{(1)} s$ and $g_{p q}^{(2)} s$ and the measurement voltage $V$ in the case of arbitrarily polarized aperture field. Then, the hypothesis of vertical or horizontal aperture field polarizations will be enforced (Section 4.1). Afterwards, the regularized SVD inversion is described (Section 4.2) and, finally, the radiation process is illustrated (Section 4.3).

4.1. Relevant Unknowns-to-Data Links. Let us begin by observing that the contribution to the radiated electric field at the measurement point $(x, y, z)$ due to the generic aperture element $\mathrm{d} x_{i} \mathrm{~d} z_{i}$ located at the point $\left(x_{i}, 0, z_{i}\right)$ in the aperture plane and corresponding to the aperture field $\underline{E}_{a}\left(x_{i}, z_{i}\right)=$ $E_{a_{x}}\left(x_{i}, z_{i}\right) \hat{i}_{x}+E_{a_{z}}\left(x_{i}, z_{i}\right) \hat{i_{z}}$ can be written as [23] $\mathrm{d} \underline{E}^{i}(x, y, z)=f(\beta, R)$

$$
\begin{aligned}
& \times\left\{-y E_{a_{x}}\left(x_{i}, z_{i}\right) \hat{i_{x}}\right. \\
&+\left[\left(z-z_{i}\right) E_{a_{z}}\left(x_{i}, z_{i}\right)-\left(x-x_{i}\right) E_{a_{x}}\left(x_{i}, z_{i}\right)\right] \hat{i}_{y} \\
&\left.\quad-y E_{a_{z}}\left(x_{i}, z_{i}\right) \hat{i}_{z}\right\} \mathrm{d} x_{i} \mathrm{~d} z_{i} \\
&=\mathrm{d} E_{x}^{i} \hat{i}_{x}+\mathrm{d} E_{y}^{i} \hat{i_{y}}+\mathrm{d} E_{z}^{i} \hat{i}_{z},
\end{aligned}
$$

where

$$
f(\beta, R)=-\frac{1}{2 \pi}\left(\frac{j \beta}{R}+\frac{1}{R^{2}}\right) \frac{e^{-j \beta R}}{R}
$$

and $R=\sqrt{\left(x-x_{i}\right)^{2}+y^{2}+\left(z-z_{i}\right)^{2}}$. 
Accounting for the probe features, that is, performing the probe compensation, should be in principle carried out by exploiting the plane wave spectrum (PWS) of the radiated field and by taking into account the spectral properties of the probe effective length. However, to unburden the approach, we assume that the probe is located in the far field of the aperture element $\mathrm{d} x_{i} \mathrm{~d} z_{i}$ so that the contribution of the latter to $V$ can be calculated as

$$
\mathrm{d} V^{i}=h_{x}\left(\vartheta_{i}, \varphi_{i}\right) \mathrm{d} E_{x}^{i}+h_{y}\left(\vartheta_{i}, \varphi_{i}\right) \mathrm{d} E_{y}^{i}+h_{z}\left(\vartheta_{i}, \varphi_{i}\right) \mathrm{d} E_{z}^{i},
$$

where $\vartheta_{i}$ and $\varphi_{i}$ are defined in Figure 2 and are such that

$$
\begin{gathered}
\cos \vartheta_{i}=\frac{\left(z-z_{i}\right)}{R}, \\
\sin \varphi_{i}=\frac{y x_{i}}{R_{1} d},
\end{gathered}
$$

$R_{1}=\sqrt{\left(x-x_{i}\right)^{2}+y^{2}}, d$ is the radius of the measurement cylinder, and $h_{x}, h_{y}$, and $h_{z}$ are the $x, y$, and $z$ components, respectively, of the probe effective length in the Oxyz reference system.

In light of (16) and (18), the voltage $V$ acquired by the probe and due to the whole aperture can be written as

$$
\begin{aligned}
V(x, y, z)=-\iint_{A} f(\beta, R) & \left\{-y E_{a_{x}}\left(x_{i}, z_{i}\right) h_{x}\left(\vartheta_{i}, \varphi_{i}\right)\right. \\
+ & {\left[\left(z-z_{i}\right) E_{a_{z}}\left(x_{i}, z_{i}\right)\right.} \\
& \left.-\left(x-x_{i}\right) E_{a_{x}}\left(x_{i}, z_{i}\right)\right] h_{y}\left(\vartheta_{i}, \varphi_{i}\right) \\
& \left.-y E_{a_{z}}\left(x_{i}, z_{i}\right) h_{z}\left(\vartheta_{i}, \varphi_{i}\right)\right\} \mathrm{d} x_{i} \mathrm{~d} z_{i},
\end{aligned}
$$

where $A=A_{1} \cup A_{2}$ and $h_{x}, h_{y}$, and $h_{z}$ can be calculated (see Appendix A) according to (A.1) and (A.2).

Equation (20) defines the operator linking the aperture field (and, thus, the coefficients $g_{p q}^{(1)} s$ and $g_{p q}^{(2)} s$ ) to the measurement voltage. In the cases of vertical and horizontal polarizations, it specifies as

$$
\begin{gathered}
V^{V}(x, y, z)=-\iint_{A} f(\beta, R)\left[\left(z-z_{i}\right) h_{y}\left(\vartheta_{i}, \varphi_{i}\right)-y h_{z}\left(\vartheta_{i}, \varphi_{i}\right)\right] \\
\quad \times E_{a_{z}}\left(x_{i}, z_{i}\right) \mathrm{d} x_{i} \mathrm{~d} z_{i}, \\
V^{H}(x, y, z)=\iint_{A} f(\beta, R)\left[y h_{x}\left(\vartheta_{i}, \varphi_{i}\right)+\left(x-x_{i}\right) h_{y}\left(\vartheta_{i}, \varphi_{i}\right)\right] \\
\times E_{a_{x}}\left(x_{i}, z_{i}\right) \mathrm{d} x_{i} \mathrm{~d} z_{i},
\end{gathered}
$$

respectively.

The components of the effective length of the probe relevant to the experimental results can be calculated according to [24] (see the Appendix A).

On using the representation (15) and on sampling $V^{V}$ or $V^{H}$ at $M_{P}$ points $\left(x_{m}, y_{m}, z_{m}\right)$ of the measurement helix, (21) or (22) can be rewritten as

$$
\underline{V}=\underline{T} \underline{g}
$$

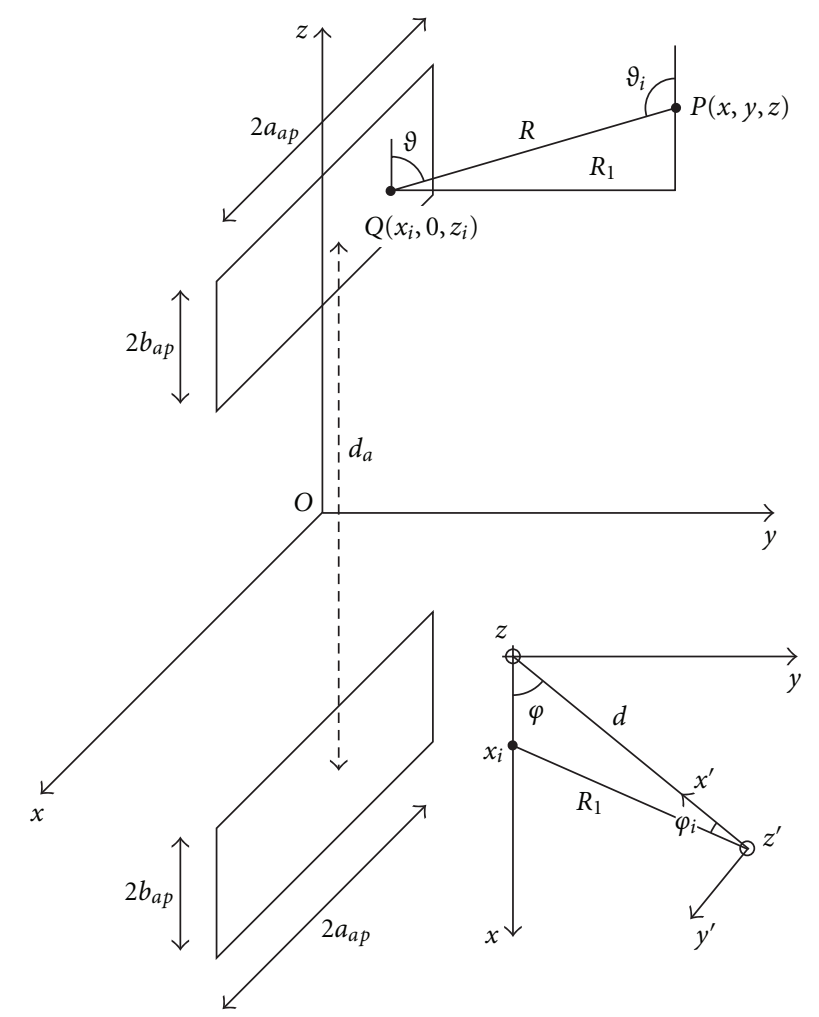

Figure 2: Relevant to the geometry of the problem.

where $\underline{V}$ is the $M_{P} \times 1$ vector containing the $M_{P}$ voltage samples $V^{V, H}\left(x_{m}, y_{m}, z_{m}\right), \underline{\underline{T}}$ is the block matrix $\left[\underline{\underline{T}}_{1}, \underline{\underline{T}}_{2}\right]$ whose generic element is detailed in Appendix B, and $g$ is the $2 P Q \times 1$ vector containing the following expansion coefficients:

$$
\begin{gathered}
g_{h}= \begin{cases}g_{p q}^{(1)} & h=(p-1) Q+q, \\
g_{p q}^{(2)} & h=P Q+(p-1) Q+q,\end{cases} \\
p=1, \ldots, P, q=1, \ldots, Q .
\end{gathered}
$$

4.2. Regularized SVD Inversion. The characterization of the antenna amounts to the determination of the projection of the aperture field $\underline{E}_{a}\left(x_{i}, z_{i}\right)$ onto the aperture plane in terms of $g$. Unfortunately, the inversion of (23) is affected by the ill-conditioning so that a regularized approach should be exploited. A possible solution is herewithin provided by the use of the truncated singular value decomposition (TSVD), even if other regularization strategies are possible [25].

4.3. Radiation. Once the components of the aperture field on the $x z$ plane have been recovered, the $\varphi$ and $\vartheta$ components of the far-field pattern $\underline{E} \infty(r, \vartheta, \varphi)$ can be determined by resorting to the PWS $\underline{\underline{E}}$ of the radiated field. More in detail, 
in the case of vertical polarization, the $\vartheta$ component is of interest, which can be determined as

$$
E_{\infty_{\vartheta}}(r, \vartheta, \varphi) \propto \frac{v}{\sqrt{1-(w / \beta)^{2}}} \widehat{E}_{z}(u, v) \frac{\mathrm{e}^{-\mathrm{j} \beta r}}{r},
$$

whereas, in the case of horizontal polarization, the $\varphi$ component of the far field is sought for, which can be determined as

$$
E_{\infty_{\varphi}}(r, \vartheta, \varphi) \propto w \hat{E}_{x}(u, v) \frac{\mathrm{e}^{-\mathrm{j} \beta r}}{r} .
$$

In (25) and (26), $u=-\beta \sin \vartheta \cos \varphi, v=\beta \cos \vartheta$, and $w=\beta \sin \vartheta \sin \varphi$.

By exploiting the Fourier transform relationship between the component $\hat{E}_{t}(u, v)$ homologous to $E_{a_{t}}, t=x, z$, having determined the expansion coefficients $g_{p q}^{(1)} \mathrm{s}$ and $g_{p q}^{(2)} \mathrm{s}$, exploiting the properties of the PSWFs $[17,18], \hat{E}_{t}(u, v)$ can be calculated as

$$
\widehat{E}_{t}(u, v)=\hat{E}_{t}^{(1)}(u, v) \mathrm{e}^{\mathrm{j} v\left(d_{a} / 2\right)}+\widehat{E}_{t}^{(2)}(u, v) \mathrm{e}^{-\mathrm{j} v\left(d_{a} / 2\right)},
$$

where

$$
\begin{aligned}
\hat{E}_{t}^{(i)}(u, v)= & -\frac{1}{2 \pi} \sum_{p=1}^{P} \sum_{q=1}^{Q} g_{p q}^{(i)} \mathrm{j}^{(p+q)} \\
& \times \sqrt{\frac{a_{a p} b_{a p}}{u^{\prime} v^{\prime}}} \sqrt{\lambda_{p}\left(c_{x}\right) \lambda_{q}\left(c_{x}\right)} \\
& \times \Phi_{p}\left[c_{x}, \frac{a_{a p}}{u^{\prime}} u\right] \Phi_{q}\left[c_{y}, \frac{b_{a p}}{v^{\prime}} v\right]
\end{aligned}
$$

and $\lambda_{i}\left(c_{w}\right)$ is the eigenvalue corresponding to the $i$ th, $1 \mathrm{D}$ PSWFs. Obviously, as an alternative to (27) and (28), $\hat{E}_{t}(u, v)$ can be evaluated by fast routines for the calculation of the Fourier transform provided that they are available.

\section{Experimental Results}

The approach described in the previous section has been experimentally validated in the anechoic chamber available at the Antenna Characterization Lab of the University of Salerno, which is provided with a NF facility system supplied by MI Technologies. The dimensions of the chamber are $8 \mathrm{~m} \times 5 \mathrm{~m} \times 4 \mathrm{~m}$. Pyramidal absorbers are positioned in order to minimize the reflections. The chamber is equipped with a vertical scanner and a rotating table, so that, by properly matching their movements, the NF data can be acquired at any point on a cylindrical surface surrounding the AUT. The vertical scanner can cover an extension of $240 \mathrm{~cm}$ and is characterized by a linear precision of $\pm 0.005 \mathrm{~cm}$. The rotating table, mounted with its rotary axis parallel to the vertical scanner, ensures an angular precision of $\pm 0.09^{\circ}$. The controller is able to simultaneously drive both the positioners. A vectorial network analyzer Anritsu 37247C provides measurements of both amplitude and phase with a wide dynamic range, high sensitivity, and linearity over

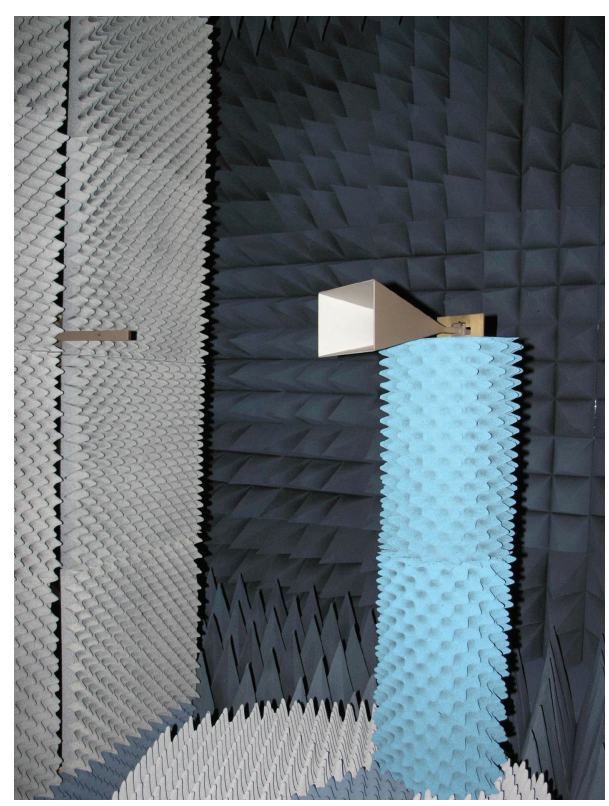

FIgURe 3: Pictorial view of the measurement system.

the range from $40 \mathrm{MHz}$ to $20 \mathrm{GHz}$. An open-ended MI6970-WR90 rectangular waveguide, whose end is tapered for minimizing the diffraction effects, is used as probe. A pictorial view of the measurement system is reported in Figure 3.

The measurements, performed at $10 \mathrm{GHz}$, refer to three test cases: a single horn with vertical polarization, a dual horn with vertical polarization for the case of sum pattern, and a dual horn with horizontal polarization for the two cases of sum and difference patterns.

The first case is considered to show the performance of the method when, at variance with [16], probe compensation is applied. The second case extends the first one to a configuration with two disconnected rectangular apertures. Finally, the third case is introduced to evaluate the performance of the approach in dependence on the polarization and on the radiated beam configuration (sum, difference).

The performance of the approach is benchmarked against the effective techniques in [6-8] based on the sampling representation of the near field, with the aim of showing how the developed approach is capable to reduce the number of required measurements, at the cost of being able to reconstruct the far-field pattern in the only $y>0$ half space. All the presented results concerning the approaches in [6-8] are probe compensated.

5.1. Single Horn. Let us consider first the case of a single $\mathrm{X}$-band, vertically polarized pyramidal horn antenna with a $0.194 \mathrm{~m} \times 0.144 \mathrm{~m}$ sized aperture. In this case, the domain $A$ consists of only one aperture.

According to the helicoidal sampling strategy described in Section 2 and relevant to the spherical AUT modelling, 1922 NF data have been acquired all around the AUT along a helix lying on a cylinder with radius $d=0.434 \mathrm{~m}$ and height $h=2.4 \mathrm{~m}$ (see Figure 1), in order to compare the here proposed approach with the experimental results in [6]. 


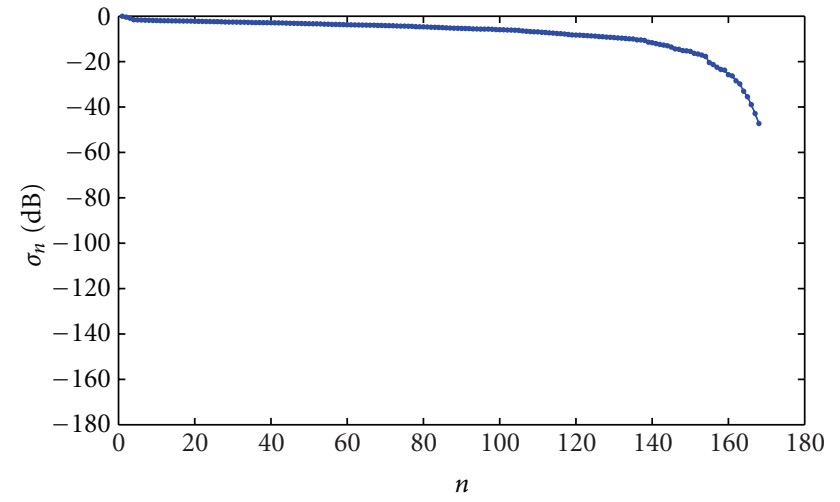

FIGURE 4: Single horn. Singular values.

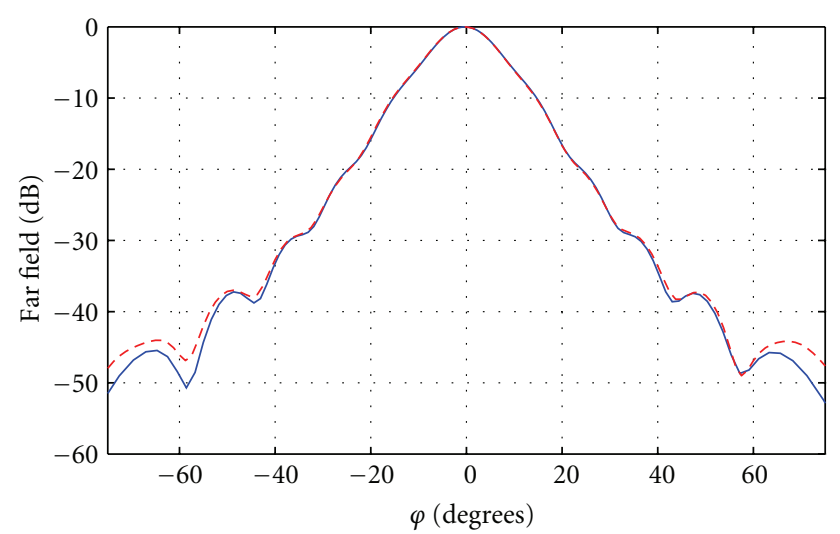

FIGURE 5: Single horn. $\varphi$ cut of the $\vartheta$ component of the far field. Blue solid line: proposed approach. Red dashed line: approach in [6].

The presented method has been applied to a set of NF data obtained from the original 1922 NF points by first rejecting all those points falling outside an azimuthal angle of $170^{\circ}$ in front of the AUT and then by decimating by a factor of two the remaining ones. A resulting set of 453 points has been exploited, and an aperture field expansion involving a number of $P \times Q=14 \times 12$ PSWFs has been considered.

Figure 4 depicts the behavior of the singular values of the matrix $T$. For this and all the other experimental test cases, the TSVD has been implemented by cutting all the singular values below a threshold set at $-40 \mathrm{~dB}$ below the maximum one. As it can be seen, only few singular values are dropped and the problem ill-conditioning is not critical for this case.

Figures 5 and 6 show $\varphi$ and $\vartheta$ cuts of the $\vartheta$ component of the far field retrieved by the proposed approach and that obtained by the approach in $[6,22]$. The agreement between the far field predicted by the approach in $[6,22]$ and direct far-field measurements has been already reported in $[6,16]$. At variance with the results shown in [16], where probe compensation was disregarded, in Figures 5 and 6, probe compensation has been applied for our approach. As can be seen, despite the significant reduction of the number of NF measurement points, the proposed approach still guarantees accurate results, when the interest is focused on the pattern radiated in the front of the AUT, as in the considered case of aperture antennas. On the contrary, the approach in $[6,22]$,

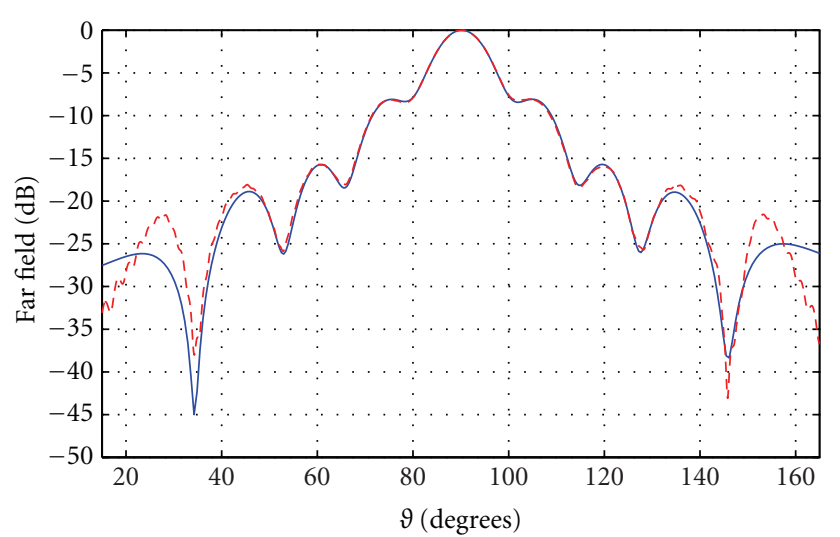

Figure 6: Single horn. $\vartheta$ cut of the $\vartheta$ component of the far field. Blue solid line: proposed approach. Red dashed line: approach in [6].

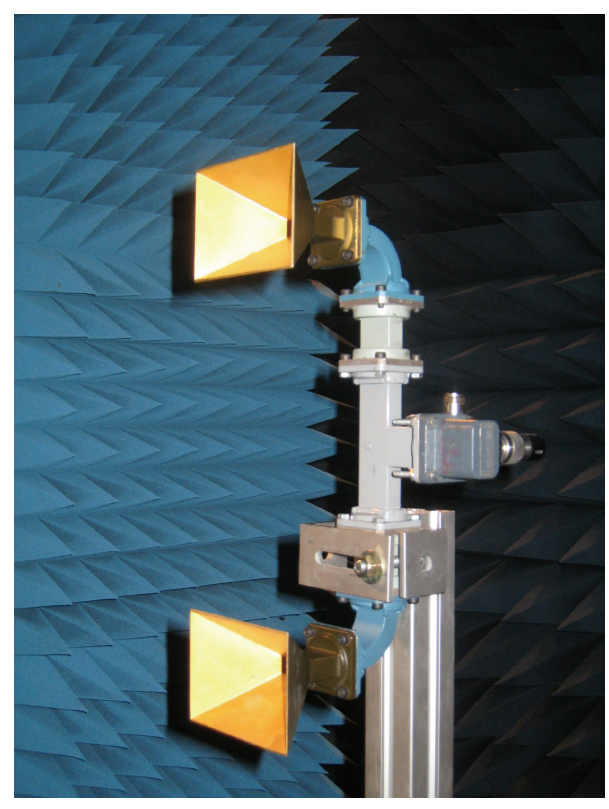

Figure 7: Photo of the vertically polarized dual horn.

even if it requires a greater number of NF data, allows the reconstruction of the complete azimuthal pattern.

5.2. Dual Horn: Vertical Polarization. Experimental results relevant to the characterization of an X-band, dual pyramidal horn antenna with vertical polarization are now illustrated.

Each horn has a $2 a_{a p} \times 2 b_{a p}=0.089 \mathrm{~m} \times 0.068 \mathrm{~m}$ sized aperture, and the centre-to-centre distance $d_{a}$ between the apertures is $0.265 \mathrm{~cm}$ (see Figure 7). The NF data have been acquired on a helix wrapping a cylinder with radius $d=0.196 \mathrm{~m}$ and height $h=2.40 \mathrm{~m}$. A number of 1946 NF samples has been collected all around the AUT to apply the technique in $[7,8]$. As before, the presented approach has been applied to the original set of NF data after having rejected all the points falling outside an azimuthal angle of $170^{\circ}$ in front of the AUT and then decimating the remaining points by a factor of 2 , thus obtaining 461 near-field samples. The aperture field expansion has comprised a number of 


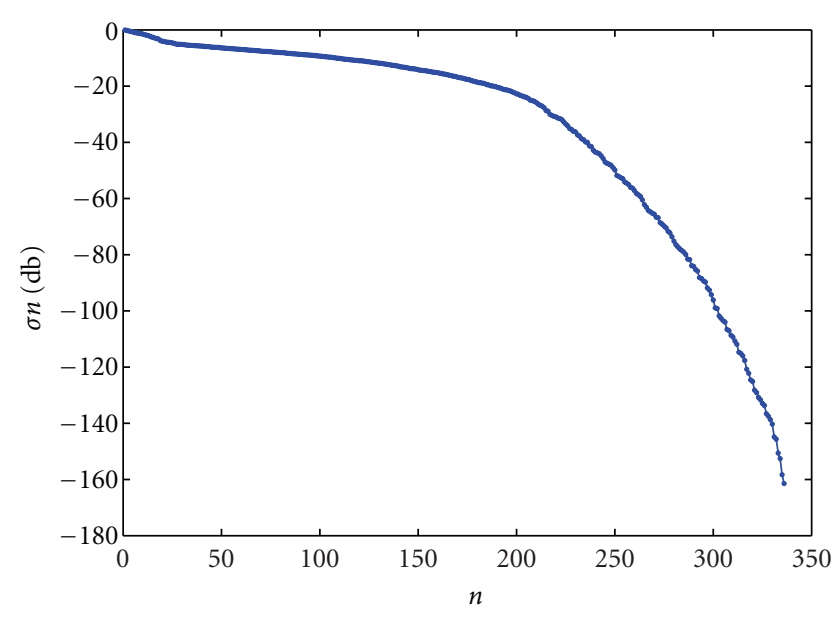

FIgURE 8: Dual horn, vertical polarization. Singular values.

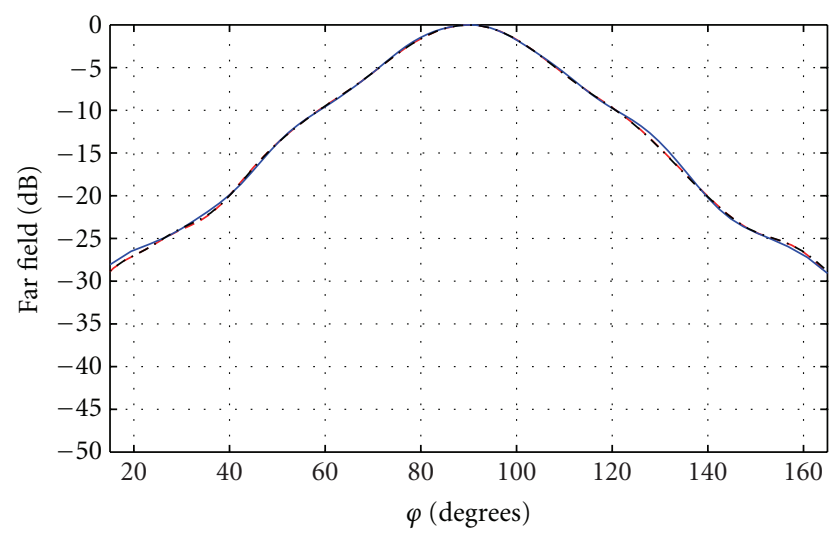

FIGURE 9: Dual horn, vertical polarization. $\varphi$ cut of the $\vartheta$ component of the far field. Blue solid line: proposed approach. Red dashed line: approach in $[7,8]$. Black dash-dot line: standard, probe compensated NF-FF transformation.

$P \times Q=12 \times 9$ PSWFs for each one of the radiating apertures. Finally, for the sake of comparisons, NF data have been acquired on the classical cylindrical grid to get the far field via the probe-compensated NF-FF transformation [13]. In this case, a number of $6440 \mathrm{NF}$ samples has been required.

Figure 8 displays the behaviour of the singular values of $\underline{\underline{T}}$ for the considered case. As it can be seen, now many more singular values fall below the $-40 \mathrm{~dB}$ threshold and so the problem ill-conditioning is more critical as it occurred for the foregoing case. This can be mainly ascribed to the mismatch between the spheroidal modelling of the source employed to determine the field sampling and the planar, disconnected modelling of the source, detailed in Section 3, and used to perform the inversions.

Figures 9 and 10 compare the $\varphi$ and $\vartheta$ cuts of the $\vartheta$ component of the far fields obtained by applying the proposed approach with those relevant to the techniques in $[7,8]$ and $[13]$. As can be seen, despite the significant reduction in the number of employed NF samples, the presented approach still guarantees accurate results when recovering the radiated field in the only $y>0$ half space.

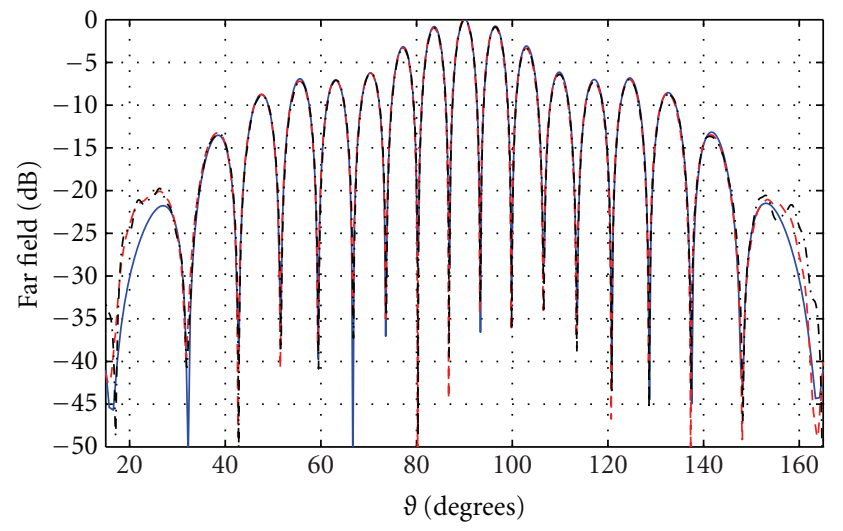

FIGURE 10: Dual horn, vertical polarization. $\vartheta$ cut of the $\vartheta$ component of the far field. Blue solid line: proposed approach. Red dashed line: approach in $[7,8]$. Black dash-dot line: standard, probecompensated NF-FF transformation.

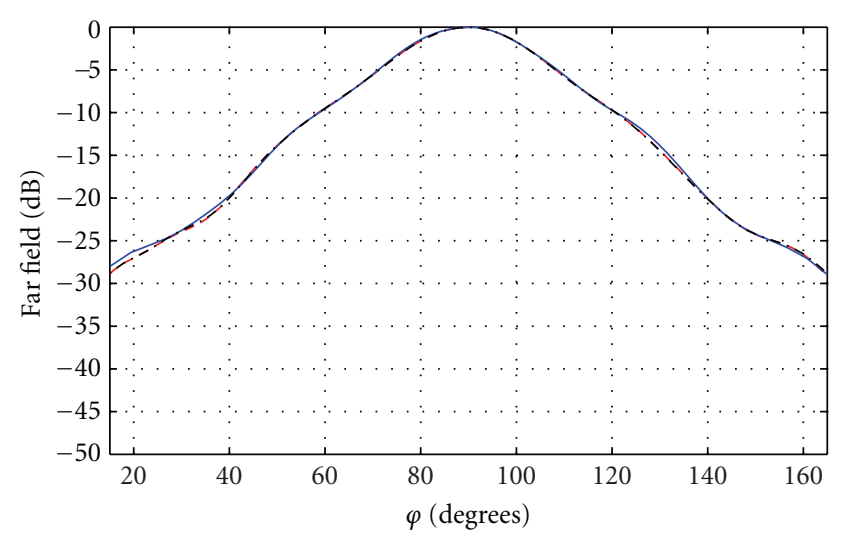

FIGURE 11: Dual horn, vertical polarization. $\varphi$ cut of the $\vartheta$ component of the far field. Blue solid line: proposed approach, when no probe-compensation is applied. Red dashed line: approach in $[7,8]$. Black dash-dot line: standard, probe compensated NF-FF transformation.

On the other side, although requiring a larger number of NF samples, the approach in $[7,8]$ allows the reconstruction of the full azimuthal pattern, but with a remarkably smaller number of samples as compared to the standard, probecompensated NF-FF transformation [13].

In order to highlight the need for compensating the receiving features of the probe, in Figures 11 and 12, we show the same results as for Figures 9 and 10, but now when our approach has been applied without any probe compensation. This can be accomplished by setting $h_{y}=$ $h_{z}=1$ in (21). As can be seen, the result worsens, almost entirely for the $\vartheta$ cut only. This can be expected since, when the probes rotate around the AUT for a fixed $z$ (and thus move along the $\varphi$ coordinate), each elementary contribution to the radiated field (see (16)) impinges on the probe itself approximately under the same azimuthal angles so that the elementary contribution to the received voltage (see (18)) experiences approximately the same receiving features of the probe (see Figure 13). On the other side, when the probe moves along the $z$ coordinate for a fixed $\varphi$, the elementary field contributions in (16) impinge with different elevation 


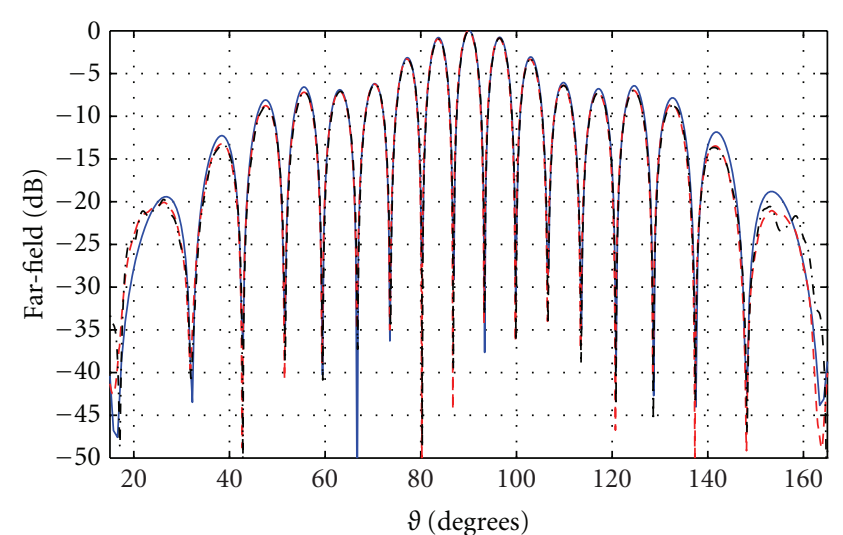

FIgURE 12: Dual horn, vertical polarization. $\vartheta$ cut of the $\vartheta$ component of the far field. Blue solid line: proposed approach, when no probe compensation is applied. Red dashed line: approach in $[7,8]$. Black dash-dot line: standard, probe-compensated NF-FF transformation.

angles so that they experience significantly different receiving features of the probe, which should be then compensated (see Figure 13).

5.3. Dual Horn: Horizontal Polarization. We finally show results concerning the characterization of an X-band, dual pyramidal horn antenna with horizontal polarization.

Each horn has a $2 a_{a p} \times 2 b_{a p}=0.068 \mathrm{~m} \times 0.089 \mathrm{~m}$ sized aperture, and the centre-to-centre distance $d_{a}$ between the apertures is $0.26 \mathrm{~cm}$. The NF data have been acquired again on a helix wrapping a cylinder with radius $d=0.196 \mathrm{~m}$ and height $h=2.40 \mathrm{~m}$. A number of $1699 \mathrm{NF}$ samples has been collected all around the AUT for the technique in $[7,8]$, whereas the presented approach has been applied to such a data set by retaining all the points within an azimuthal angle of $170^{\circ}$ in front of the AUT and then decimating them by a factor of 2, obtaining $399 \mathrm{NF}$ samples. A number of $P \times Q=9 \times 12$ PSWFs for each one of the radiating apertures has been searched for.

The two cases of a sum pattern and of a difference pattern have been dealt with. They have been obtained by setting "inphase" and "out-of-phase" the aperture fields by properly exploiting a Magic-Tee.

Figures 14 and 15 compare the $\varphi$ and $\vartheta$ cuts, respectively, of the $\varphi$ component of the far fields obtained by the approach in $[7,8]$ and by the presented one in the case of sum pattern. The results obtained by the approach in $[7,8]$ have been already validated for this case in [8] also via direct FF measurements. As it can be seen, again despite the significant reduction in the number of employed NF samples, the presented approach still guarantees accurate results in recovering the radiated field in the only $y>0$ half space. For reader's convenience, Figure 16 shows the amplitude and phase of the aperture field for the domain $A=A_{1} \cup A_{2}$ which appears to be "in-phase," and Figure 17 shows a 2D image of the $\varphi$ component of the radiated field in the spectral, $u v$ plane.

Finally, Figure 18 shows the good agreement between the $\vartheta$ cut of the $\varphi$ component of the far fields obtained by the two compared approaches in the case of difference pattern, where the results obtained by the approach in $[7,8]$ have been already validated in [8] for this case. The $\varphi$ cut is not shown since it is obviously vanishing. Furthermore, Figure 19 displays the amplitude and phase of the aperture field for the domain $A=A_{1} \cup A_{2}$ which now appears to be "out-of-phase," whereas Figure 20 shows a 2D image of the $\varphi$ component of the radiated field in the spectral, $u v$ plane.

\section{Conclusions and Future Developments}

In this paper, a new NF-FF transformation technique for the characterization of aperture antennas in a cylindrical geometry has been proposed. The approach exploits a fast scanning strategy based on a "nonredundant sampling representation" of the near field and on synchronous movements of probe and AUT. It allows the characterization of aperture antennas, even with disconnected apertures, and conveniently exploits the available a priori information on the AUT shape and size. The technique performs also a proper compensation of the probe effects and exploits a regularized version of the SVD approach.

The experimental analysis performed on single or dual horn antennas, involving different aperture field polarizations and beam configurations (sum, difference), and the comparisons with the effective approach in $[6,8]$ have highlighted that the proposed technique requires a significantly lower number of NF samples to guarantee an accuracy comparable to that in $[6,8]$, when the interest is focused on the pattern radiated in the half space in front of the AUT. Of course, the approach in $[6,8]$, even if in need for a greater number of NF data, allows the reconstruction of the full azimuthal pattern.

Future developments of the presented technique regard extensions to

(i) different scanning configurations, for example, planar spiral scanning [26];

(ii) different shapes for the AUT apertures (e.g., circular [27]).

\section{Appendices}

\section{A. Probe Compensation Formulas}

The components $h_{x}, h_{y}$, and $h_{z}$ can be related to the Cartesian components $h_{x^{\prime}}, h_{y^{\prime}}$, and $h_{z^{\prime}}$ of the probe effective length in the $O x^{\prime} y^{\prime} z^{\prime}$ probe reference system as (Figure 2)

$$
\begin{gathered}
h_{x}\left(\vartheta_{i}, \varphi_{i}\right)=-h_{x^{\prime}}\left(\vartheta_{i}, \varphi_{i}\right) \cos \varphi+h_{y^{\prime}}\left(\vartheta_{i}, \varphi_{i}\right) \sin \varphi, \\
h_{y}\left(\vartheta_{i}, \varphi_{i}\right)=-h_{x^{\prime}}\left(\vartheta_{i}, \varphi_{i}\right) \sin \varphi-h_{y^{\prime}}\left(\vartheta_{i}, \varphi_{i}\right) \cos \varphi, \\
h_{z}\left(\vartheta_{i}, \varphi_{i}\right)=h_{z^{\prime}}\left(\vartheta_{i}, \varphi_{i}\right),
\end{gathered}
$$

where $\cos \varphi=x / d, \sin \varphi=y / d$, and the $O x^{\prime} y^{\prime} z^{\prime}$ probe reference system is understood to be oriented so that the probe aperture is centered in the $y^{\prime} z^{\prime}$ plane, while the $x^{\prime}$ axis points towards the $z$ axis. The components $h_{x^{\prime}}, h_{y^{\prime}}$, and $h_{z^{\prime}}$, 


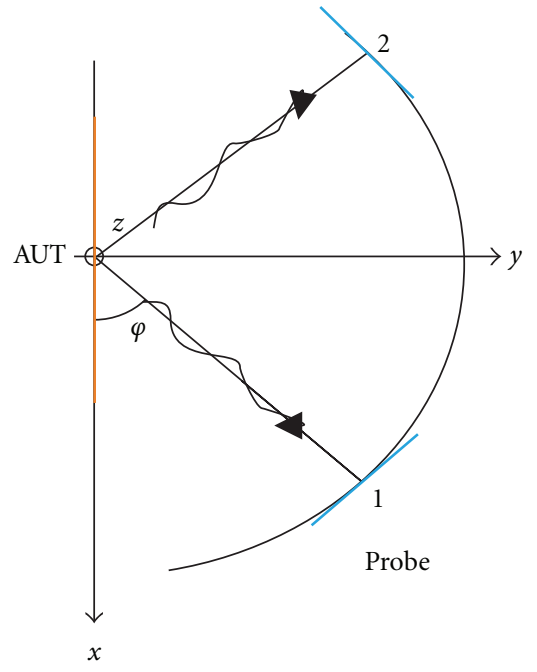

(a)

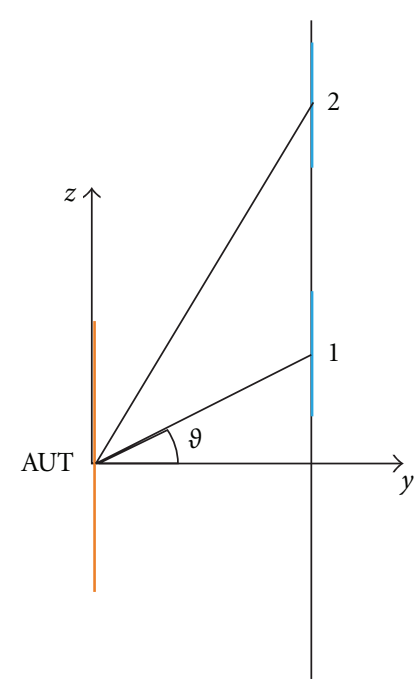

(b)

FIGURE 13: Illustrating the need for probe compensation. When the probe rotates around the AUT from position 1 to 2 (a), approximately the same receiving features of the probe are involved. When the probe moves along the $z$ axis from position 1 to 2 (b), different receiving features of the probe become relevant.

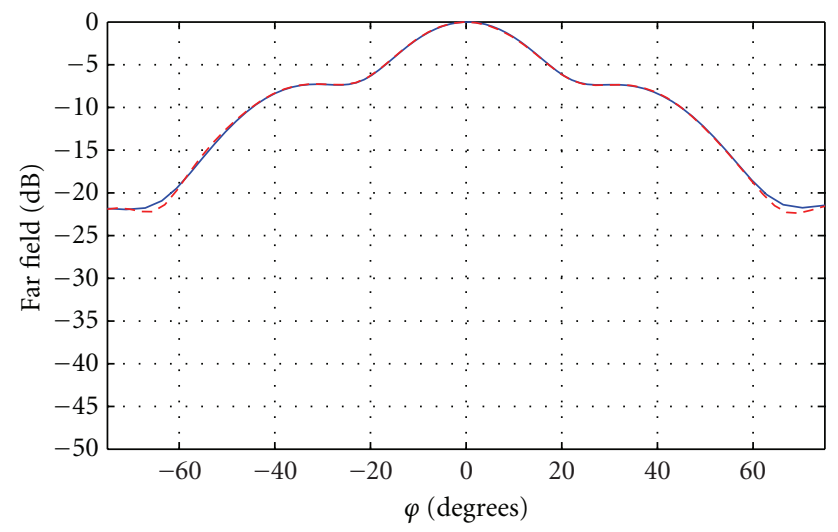

FIGURE 14: Dual horn, horizontal polarization, sum pattern. $\varphi$ cut of the $\varphi$ component of the far field. Blue solid line: proposed approach. Red dashed line: approach in $[7,8]$.

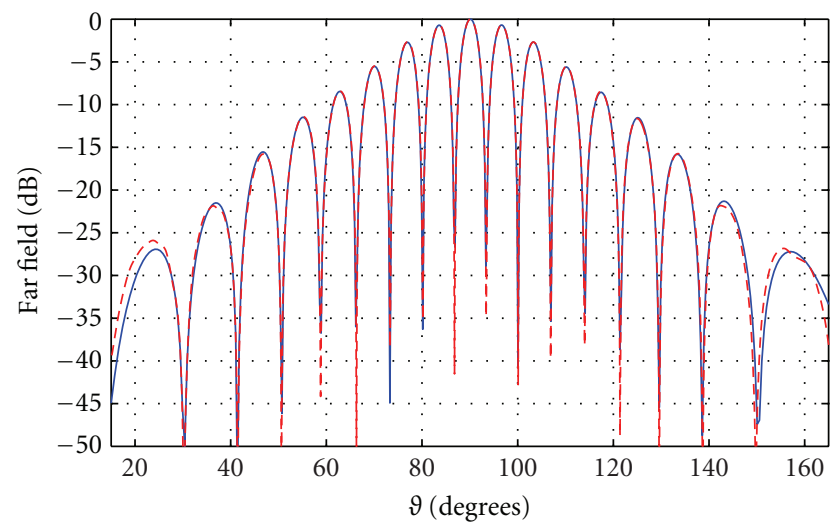

FIGURE 15: Dual horn, horizontal polarization, sum pattern. $\vartheta$ cut of the $\varphi$ component of the far field. Blue solid line: proposed approach. Red dashed line: approach in $[7,8]$.

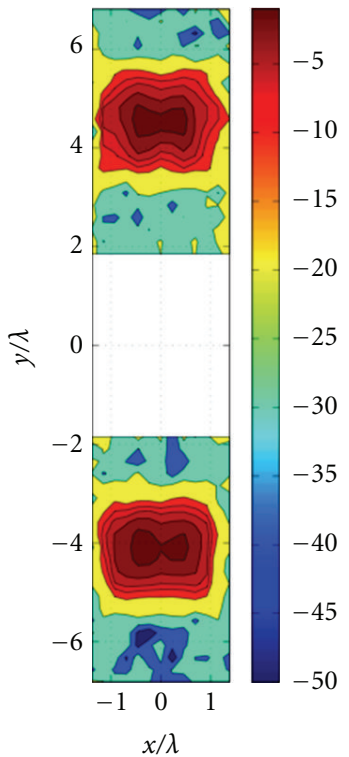

(a)

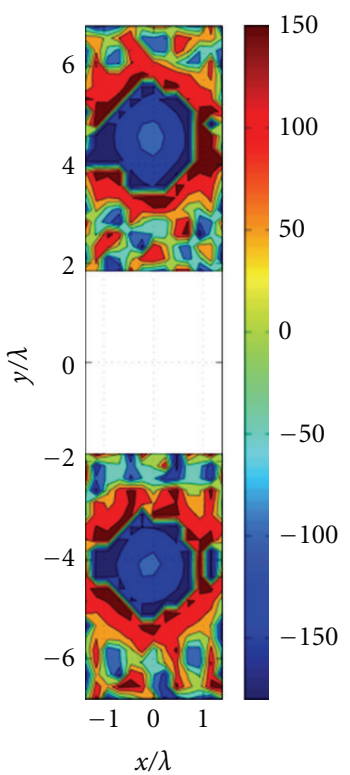

(b)
Figure 16: Dual horn, horizontal polarization, sum pattern. (a) amplitude of the aperture field distribution. (b) phase of the aperture field distribution.

in turn, can be linked to the $\vartheta^{\prime}$ and $\varphi^{\prime}$ components $h_{\vartheta^{\prime}}$ and $h_{\varphi^{\prime}}$ as

$$
\begin{gathered}
h_{x^{\prime}}\left(\vartheta_{i}, \varphi_{i}\right)=h_{\vartheta^{\prime}}\left(\vartheta_{i}, \varphi_{i}\right) \cos \vartheta_{i} \cos \varphi_{i}-h_{\varphi^{\prime}}\left(\vartheta_{i}, \varphi_{i}\right) \sin \varphi_{i}, \\
h_{y^{\prime}}\left(\vartheta_{i}, \varphi_{i}\right)=h_{\vartheta^{\prime}}\left(\vartheta_{i}, \varphi_{i}\right) \cos \vartheta_{i} \sin \varphi_{i}+h_{\varphi^{\prime}}\left(\vartheta_{i}, \varphi_{i}\right) \cos \varphi_{i}, \\
h_{z^{\prime}}\left(\vartheta_{i}, \varphi_{i}\right)=-h_{\vartheta^{\prime}}\left(\vartheta_{i}, \varphi_{i}\right) \sin \vartheta_{i} .
\end{gathered}
$$

The probe employed in this paper for the measurements is an $a \times b$ sized open-ended rectangular waveguide [24]. 


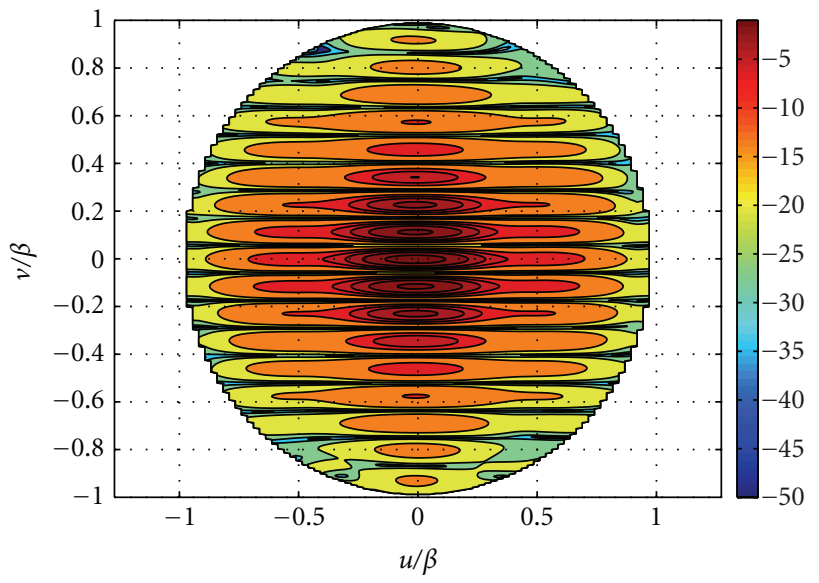

FIgURE 17: Dual horn, horizontal polarization, sum pattern. Amplitude of the $\varphi$ component of the far field.

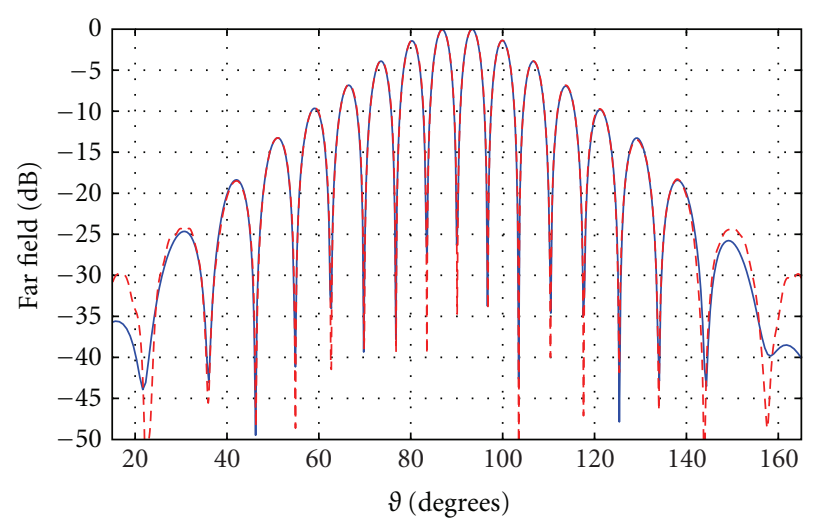

FIGURE 18: Dual horn, horizontal polarization, difference pattern. $\vartheta$ cut of the $\varphi$ component of the far field. Blue solid line: proposed approach. Red dashed line: approach in $[7,8]$.

The dimensionless components $h_{\vartheta_{S}}$ and $h_{\varphi_{S}}$ of the probe effective length in the $O x_{s} y_{s} z_{s}$ reference system, the same employed in [24], are

$$
\begin{aligned}
h_{\vartheta_{S}}\left(\vartheta_{S}, \varphi_{S}\right)= & \left\{\frac{4}{\pi^{2}}\left[1+\left(\frac{k_{T E_{10}}}{\beta}\right)+\Gamma\left(1-\left(\frac{k_{T E_{10}}}{\beta}\right)\right)\right]+C_{0}\right\} \\
& \times \sin \varphi_{S} \frac{1+\left(k_{T E_{10}} / \beta\right) \cos \vartheta_{S}}{1+\left(k_{T E_{10}} / \beta\right)} \frac{\sin \left(\beta(b / 2) \sin \vartheta_{S}\right)}{\beta(b / 2) \sin \vartheta_{S}}, \\
h_{\varphi_{S}}\left(\vartheta_{S}, \varphi_{S}\right)= & \cos \varphi_{S} \cos \left(\beta\left(\frac{a}{2}\right) \sin \vartheta_{S}\right) \\
& \times\left[\frac{\cos \vartheta_{S}+\left(k_{T E_{10}} / \beta\right)+\Gamma\left(\cos \vartheta_{S}-\left(k_{T E_{10}} / \beta\right)\right)}{(\pi / 2)^{2}-\left(\beta(a / 2) \sin \vartheta_{S}\right)^{2}}\right. \\
& \left.+C_{0}\right]
\end{aligned}
$$

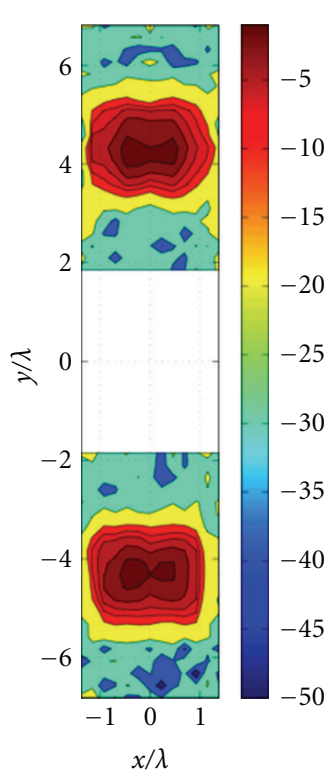

(a)

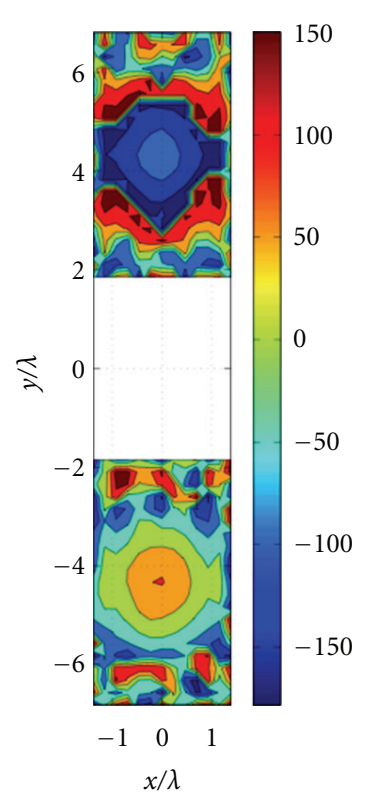

(b)
FIGURE 19: Dual horn, horizontal polarization, difference pattern. (a) amplitude of the aperture field distribution. (b) phase of the aperture field distribution.

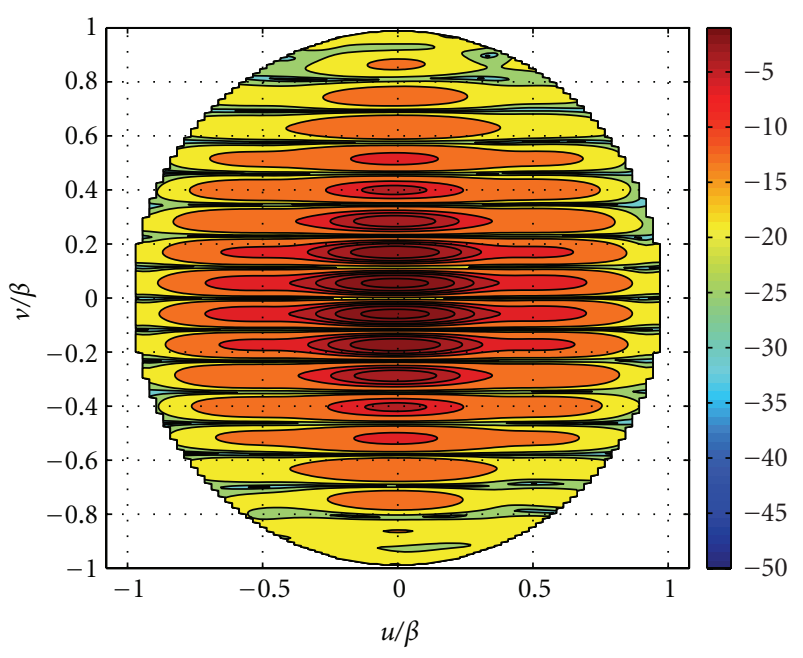

FIGURE 20: Dual horn, horizontal polarization, difference pattern. Amplitude of the $\varphi$ component of the far field.

where $\vartheta_{S}$ and $\varphi_{S}$ are the $\vartheta$ and $\varphi$ coordinates of the corresponding reference system, $k_{T E_{10}}$ is the propagation constant of the $T E_{10}$ propagation mode of the $a \times b$ sized rectangular waveguide, and $\Gamma$ is the reflection coefficient of the $T E_{10}$ mode from the end of the waveguide, whose value at $10 \mathrm{GHz}$ has been measured in [24] and is approximately equal to $0.0603+j 0.2837$. Moreover, $C_{0}$ is a real constant whose value (0.129) has been numerically evaluated according to [24].

During the scanning, the probe is oriented so that the electric field polarization for the $T E_{10}$ mode matches the polarization of the aperture field of interest for the AUT (i.e., vertical or horizontal). 


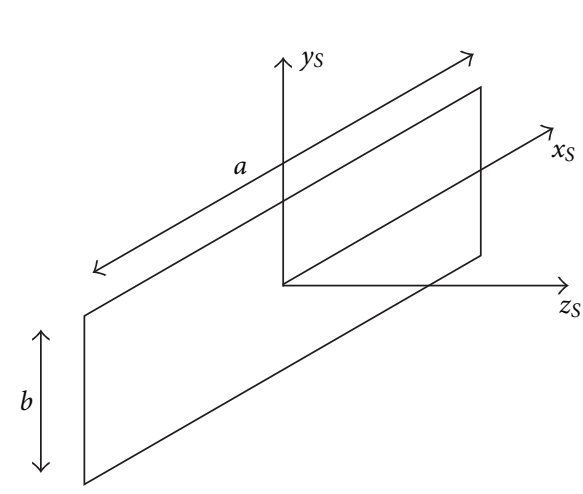

(a)

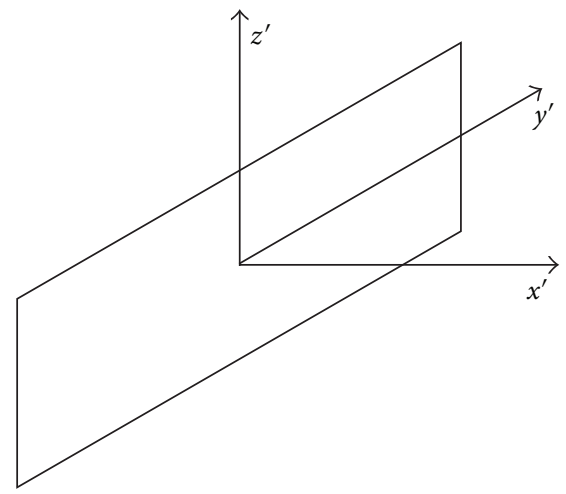

(b)

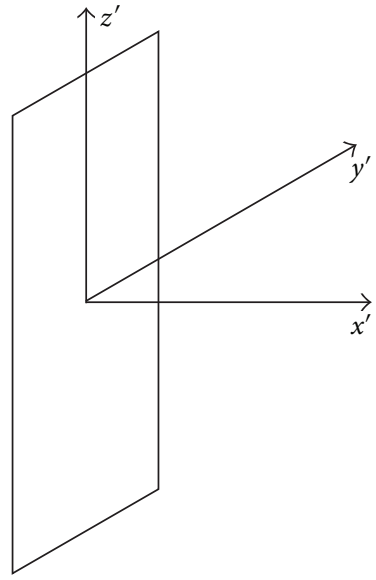

(c)

FIGURE 21: Reference systems relevant to the probe compensation. (a) Yaghjian's. (b) vertical polarization. (c) horizontal polarization.

Accordingly, in the case of vertical polarization, $x_{S}=y^{\prime}$, $y_{S}=z^{\prime}$, and $z_{S}=x^{\prime}$ (see Figure 21). Consequently,

$$
\begin{gathered}
\tan \varphi_{S}=\frac{1}{\tan \vartheta_{i} \sin \varphi_{i}}, \\
\cos \vartheta_{S}=\sin \vartheta_{i} \cos \varphi_{i} \\
h_{\vartheta^{\prime}}\left(\vartheta_{i}, \varphi_{i}\right)=-h_{\vartheta_{S}}\left(\vartheta_{S}, \varphi_{S}\right) \cos \varphi_{i} \sin \varphi_{S}-h_{\varphi_{S}}\left(\vartheta_{S}, \varphi_{S}\right) \frac{\cos \varphi_{S}}{\sin \vartheta_{i}} \\
h_{\varphi^{\prime}}\left(\vartheta_{i}, \varphi_{i}\right)=h_{\vartheta_{S}}\left(\vartheta_{S}, \varphi_{S}\right) \frac{\cos \varphi_{S}}{\sin \vartheta_{i}}-h_{\varphi_{S}}\left(\vartheta_{S}, \varphi_{S}\right) \cos \varphi_{i} \sin \varphi_{S}
\end{gathered}
$$

On the other side, in the case of horizontal polarization, $x_{S}=-z^{\prime}, y_{S}=y^{\prime}$, and $z_{S}=x^{\prime}$. Thus,

$$
\begin{aligned}
\tan \varphi_{S}= & -\tan \vartheta_{i} \sin \varphi_{i}, \\
\cos \vartheta_{S}= & \sin \vartheta_{i} \cos \varphi_{i}, \\
h_{\vartheta^{\prime}}\left(\vartheta_{i}, \varphi_{i}\right)= & h_{\vartheta_{S}}\left(\vartheta_{S}, \varphi_{S}\right) \cos \varphi_{S} \cos \varphi_{i} \\
& +h_{\varphi_{S}}\left(\vartheta_{S}, \varphi_{S}\right)\left[\cos \varphi_{S} \cos \vartheta_{i} \sin \varphi_{i}-\sin \varphi_{S} \sin \vartheta_{i}\right], \\
h_{\varphi^{\prime}}\left(\vartheta_{i}, \varphi_{i}\right)= & h_{\vartheta_{S}}\left(\vartheta_{S}, \varphi_{S}\right)\left[\sin \varphi_{S} \sin \vartheta_{i}-\cos \varphi_{S} \cos \vartheta_{i} \sin \varphi_{i}\right] \\
& +h_{\varphi_{S}}\left(\vartheta_{S}, \varphi_{S}\right) \cos \varphi_{S} \cos \varphi_{i} .
\end{aligned}
$$

\section{B. The Matrix $\underline{\underline{T}}$}

The explicit expression of the generic element of the block matrix $\underline{\underline{T}}=\left[\underline{\underline{T}}_{1}, \underline{\underline{T}}_{2}\right]$ is given by

$$
\begin{gathered}
T_{1_{m h}}^{V}=-\iint_{A} f\left(\beta, R_{m}\right)\left[\left(z_{m}-z_{i}\right) h_{y}\left(\vartheta_{i}, \varphi_{i}\right)-y_{m} h_{z}\left(\vartheta_{i}, \varphi_{i}\right)\right] \\
\times \Phi_{p}\left[c_{x}, x_{i}\right] \Phi_{q}\left[c_{z}, z_{i}-\frac{d_{a}}{2}\right] \mathrm{d} x_{i} \mathrm{~d} z_{i},
\end{gathered}
$$

$$
\begin{gathered}
T_{1_{m h}}^{H}=\iint_{A} f\left(\beta, R_{m}\right)\left[y_{m} h_{x}\left(\vartheta_{i}, \varphi_{i}\right)+\left(x_{m}-x_{i}\right) h_{y}\left(\vartheta_{i}, \varphi_{i}\right)\right] \\
\times \Phi_{p}\left[c_{x}, x_{i}\right] \Phi_{q}\left[c_{z}, z_{i}-\frac{d_{a}}{2}\right] \mathrm{d} x_{i} \mathrm{~d} z_{i}, \\
T_{2_{m h}}^{V}=-\iint_{A} f\left(\beta, R_{m}\right)\left[\left(z_{m}-z_{i}\right) h_{y}\left(\vartheta_{i}, \varphi_{i}\right)-y_{m} h_{z}\left(\vartheta_{i}, \varphi_{i}\right)\right] \\
\quad \times \Phi_{p}\left[c_{x}, x_{i}\right] \Phi_{q}\left[c_{z}, z_{i}+\frac{d_{a}}{2}\right] \mathrm{d} x_{i} \mathrm{~d} z_{i}, \\
T_{2_{m h}}^{H}=\iint_{A} f\left(\beta, R_{m}\right)\left[y_{m} h_{x}\left(\vartheta_{i}, \varphi_{i}\right)+\left(x_{m}-x_{i}\right) h_{y}\left(\vartheta_{i}, \varphi_{i}\right)\right] \\
\times \Phi_{p}\left[c_{x}, x_{i}\right] \Phi_{q}\left[c_{z}, z_{i}+\frac{d_{a}}{2}\right] \mathrm{d} x_{i} \mathrm{~d} z_{i},
\end{gathered}
$$

with $h=(p-1) Q+q, p=1, \ldots, P, q=1, \ldots, Q$, and $R_{m}=$ $\sqrt{\left(x_{m}-x_{i}\right)^{2}+y_{m}^{2}+\left(z_{m}-z_{i}\right)^{2}}$.

Obviously, the matrices $\underline{T}_{1}$ and $\underline{T}_{2}$ are constructed by considering only the terms in (B.1) referring to the polarization of interest (i.e., only the terms with the $V$ or $H$ superscripts).

\section{References}

[1] A. D. Yaghjian, "An overview of near-field antenna measurements," IEEE Transactions on Antennas and Propagation, vol. AP-34, no. 1, pp. 30-45, 1986.

[2] "Special Issue on near-field scanning techniques," IEEE Transactions on Antennas and Propagation, vol. AP-36, no. 6, pp. 727-901, 1988.

[3] G. E. Evans, Antenna Measurement Techniques, Artech House, Boston, Mass, USA, 1990.

[4] C. A. Balanis, Modern Antenna Handbook, John Wiley \& Sons, Hoboken, NJ, USA, 2008.

[5] O. M. Bucci, C. Gennarelli, G. Riccio, and C. Savarese, "Nonredundant NF-FF transformation with helicoidal scanning," Journal of Electromagnetic Waves and Applications, vol. 15, no. 11, pp. 1507-1519, 2001. 
[6] F. D’Agostino, F. Ferrara, C. Gennarelli, R. Guerriero, and M. Migliozzi, "Experimental results validating the near-field to far-field transformation technique with helicoidal scan," Open Electrical and Electronic Engineering Journal, vol. 4, pp. 10-15, 2010.

[7] F. D'Agostino, F. Ferrara, C. Gennarelli, R. Guerriero, and M. Migliozzi, "Near-field-far-field transformation technique with helicoidal scanning for elongated antennas," Progress in Electromagnetics Research, vol. 4, pp. 249-261, 2008.

[8] F. D'Agostino, F. Ferrara, C. Gennarelli, R. Guerriero, and M. Migliozzi, "Laboratory tests assessing the effectiveness of the NF-FF transformation with helicoidal scanning for electrically long antennas," Progress in Electromagnetics Research, vol. 98, pp. 375-388, 2009.

[9] F. D’Agostino, F. Ferrara, J. A. Fordham et al., "An effective near-field-far-field transformation technique for elongated antennas using a fast helicoidal scan," IEEE Antennas and Propagation Magazine, vol. 51, no. 4, pp. 134-141, 2009.

[10] R. G. Yaccarino, L. I. Williams, and Y. Rahmat-Samii, "Linear spiral sampling for the bipolar planar near-field antenna measurement technique," IEEE Transactions on Antennas and Propagation, vol. 44, no. 7, pp. 1049-1051, 1996.

[11] O. M. Bucci, C. Gennarelli, and C. Savarese, "Representation of electromagnetic fields over arbitrary surfaces by a finite and nonredundant number of samples," IEEE Transactions on Antennas and Propagation, vol. 46, no. 3, pp. 351-359, 1998.

[12] W. M. Leach Jr. and D. T. Paris, "Probe compensated near-field measurements on a cylinder," IEEE Transactions on Antennas and Propagation, vol. AP-21, no. 4, pp. 435-445, 1973.

[13] A. D. Yaghjian, "Near-field antenna measurement on a cylindrical surface: a source scattering matrix formulation," Tech. Rep. 696, Government Printing Office, Washington, DC, USA, 1977.

[14] A. Capozzoli, C. Curcio, G. D’Elia, and A. Liseno, "Phaseless antenna characterization by effective aperture field and data representations," IEEE Transactions on Antennas and Propagation, vol. 57, no. 1, pp. 215-230, 2009.

[15] A. Capozzoli, C. Curcio, G. D’Elia, and A. Liseno, "Singularvalue optimization in plane-polar near-field antenna characterization," IEEE Antennas and Propagation Magazine, vol. 52, no. 2, pp. 103-112, 2010.

[16] A. Capozzoli, C. Curcio, G. D’Elia et al., "A SVD-based approach to helicoidal NF-FF transformations," in Proceedings of the 4th European Conference on Antennas and Propagation (EuCAP '10), pp. 1-5, Barcelona, Spain, April 2010.

[17] B. R. Frieden, "Evaluation, design and extrapolation methods for optical signals, based on use of the prolate functions," in Progress in Optics, E. Wolf, Ed., vol. 9, pp. 311-407, NorthHolland, Amsterdam, The Netherlands, 1971.

[18] H. J. Landau and H. O. Pollack, "Prolate spheroidal wave functions, Fourier analysis and uncertainty-III: the dimension of the space of essentially time- and band-limited signals," Bell System Technical Journal, vol. 41, pp. 1295-1336, 1962.

[19] O. M. Bucci, G. D’Elia, F. Soldovieri, and R. Pierri, "An effective strategy in the planar near-field far-field transformation for focussing antennas," in Proceedings of the 20th ESTEC Antenna Workshop on Millimetre Wave Antenna Technology and Antenna Measurement (ESTEC '97), pp. 335342, Noordwijk, The Netherlands, June 1997.

[20] O. M. Bucci, G. D’Elia, and M. D. Migliore, “Advanced field interpolation from plane-polar samples: experimental verification," IEEE Transactions on Antennas and Propagation, vol. 46, no. 2, pp. 204-210, 1998.
[21] F. D’Agostino, F. Ferrara, C. Gennarelli, R. Guerriero, and M. Migliozzi, "The unified theory of near-field-far-field transformations with spiral scannings for nonspherical antennas," Progress In Electromagnetics Research B, vol. 14, pp. 449-477, 2009.

[22] F. D’Agostino, C. Gennarelli, G. Riccio, and C. Savarese, “Theoretical foundations of near-field-far-field transformations with spiral scannings," Progress in Electromagnetics Research, vol. 61, pp. 193-214, 2006.

[23] J. W. Goodman, Introduction to Fourier Optics, McGraw-Hill, New York, NY, USA, 1996.

[24] A. D. Yaghiian, "Approximate formulas for the far field and gain of open-ended rectangular waveguide," IEEE Transactions on Antennas and Propagation, vol. AP-32, no. 4, pp. 378-384, 1984.

[25] M. Bertero and P. Boccacci, Introduction to Inverse Problems in Imaging, Institute of Physics Publishing, Bristol, UK, 1998.

[26] F. D’Agostino, F. Ferrara, C. Gennarelli, R. Guerriero, and M. Migliozzi, "An effective NF-FF transformation technique with planar spiral scanning tailored for quasi-planar antennas," IEEE Transactions on Antennas and Propagation, vol. 56, no. 9, pp. 2981-2987, 2008.

[27] D. Slepian, "Prolate spheroidal wave functions, Fourier analysis and uncertainty-IV: extensions to many dimensions; generalized prolate spheroidal functions," Bell System Technical Journal, vol. 43, pp. 3009-3057, 1964. 

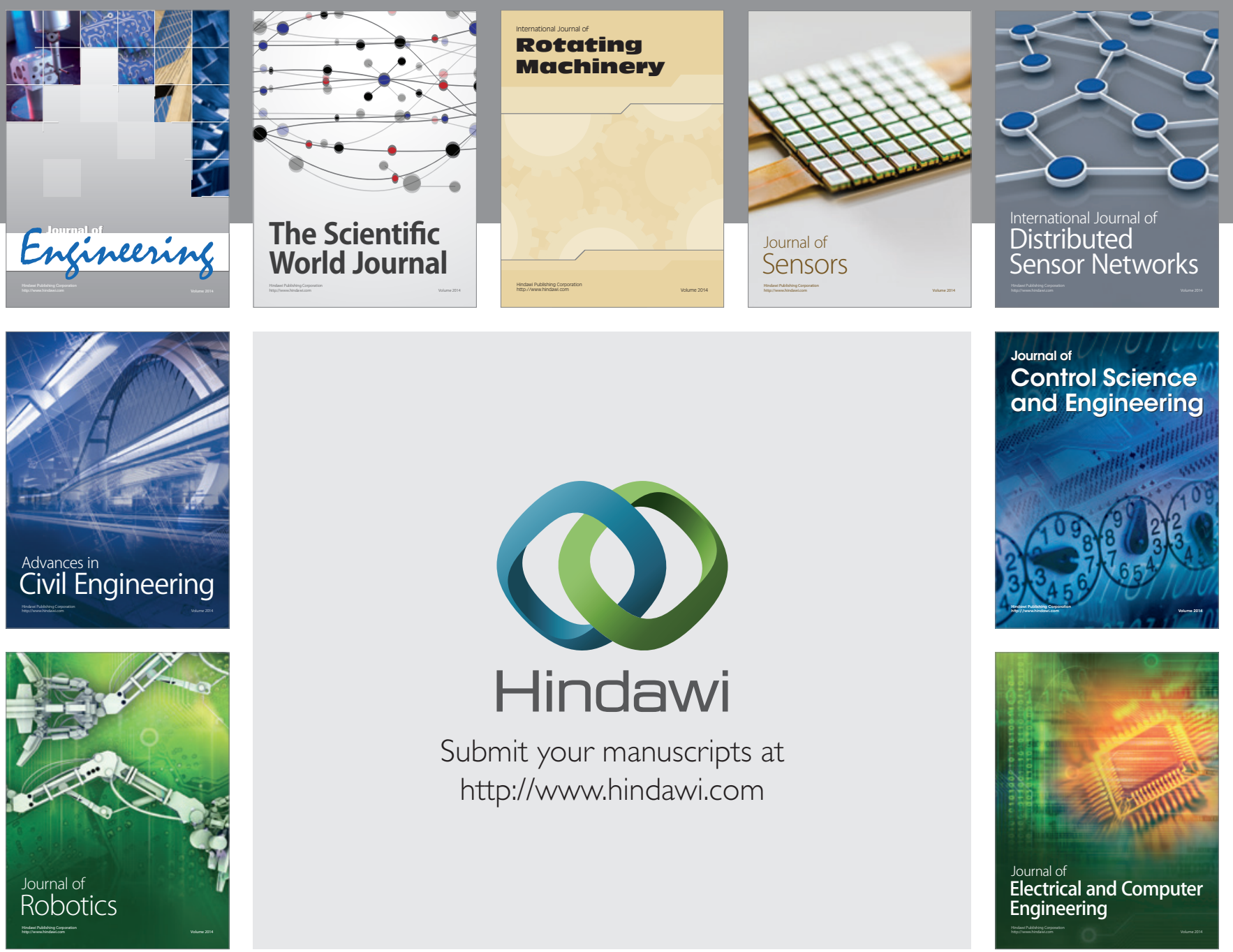

Submit your manuscripts at

http://www.hindawi.com
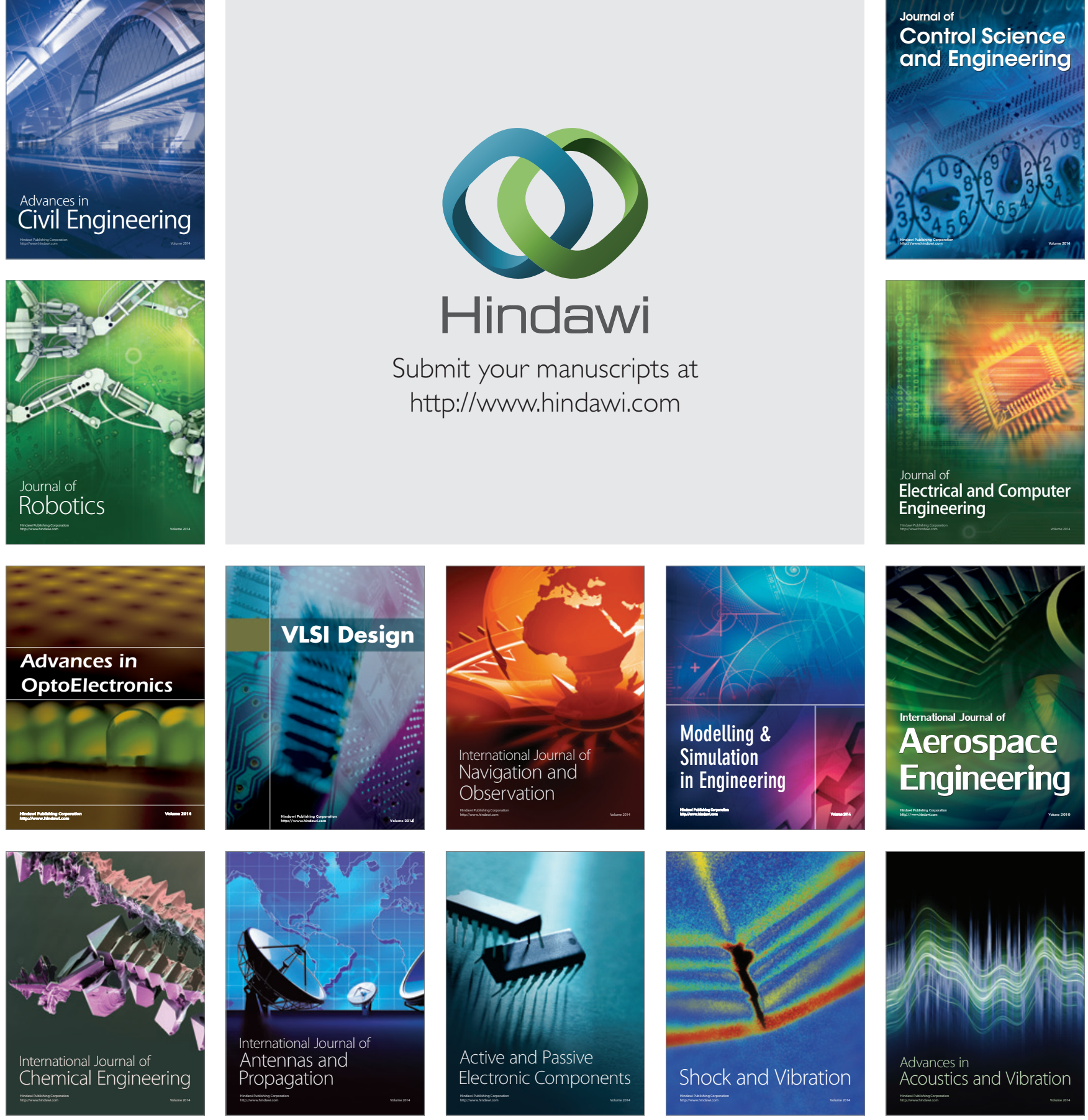